\title{
ANÁLISIS DE LA ESTRUCTURA ORGANIZACIONAL DEL CENTRO DE REINSERCIÓN SOCIAL DE TABASCO (CREST)
}

\section{ANALYSIS OF THE ORGANIZATIONAL STRUCTURE OF THE SOCIAL READAPTATION CENTER IN TABASCO}

\begin{abstract}
María Belém Banda Izeta*, Herminia Banda Izeta**, Hilda Guillemín Calderón**,

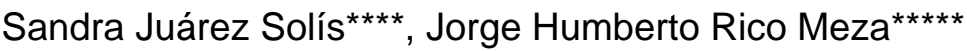

\begin{abstract}
*Alumna de la Maestría en Derecho. División Académica de Ciencias Sociales y Humanidades. Email: mabel-baniz@hotmail.com. ORCID: http://orcid.org/0000-0001-9861-0023.

**Doctora en Educación Internacional. División Académica de Ciencias Económico Administrativas. Universidad Juárez Autónoma de Tabasco. Email: hbi05@hotmail.com. ORCID: http://orcid.org/0000-00034565-3577.

***Maestra en Docencia. División Académica de Ciencias Económico Administrativas. Universidad Juárez Autónoma de Tabasco. Email: hguillemin@hotmail.com. ORCID: http://orcid.org/0000-0002-3679-8857.

****Doctora en Educación. División Académica de Ciencias Económico Administrativas. Universidad Juárez Autónoma de Tabasco. Email: marketingvhsa@outlook.com. ORCID: http://orcid.org/0000-0002-78833622.

*****Maestro en Administración Pública. División Académica de Ciencias Económico Administrativas. Universidad Juárez Autónoma de Tabasco. Email: rico_meza@hotmail.com. ORCID: http://orcid.org/00000003-1310-3677.
\end{abstract}

Dirección para recibir correspondencia: hbi05@hotmail.com

Fecha de recibido: 10 de noviembre de 2019

Fecha de aceptación: 15 de diciembre de 2019

BANDA-IZETA M.B, BANDA-IZETA H., GUILLEMÍN-CALDERÓN H., JUÁREZ-SOLÍS S. RICO-MEZA J. H. 
ANÁLISIS DE LA ESTRUCTURA ORGANIZACIONAL DEL CENTRO DE REINSERCIÓN SOCIAL DE TABASCO (CREST).

\section{RESUMEN}

OBJETIVO: Analizar la Estructura Organizacional del Centro de Reinserción Social de Tabasco (CREST).

MATERIAL Y MÉTODO: La investigación se desarrolla en una Institución dependiente de la administración pública. En un primer momento se entrevista al titular de la dependencia. En un segundo momento se entrevista al personal administrativo. Para todos los entrevistados, se utilizó una entrevista estructurada con la finalidad de obtener la información definida en sus actividades que posteriormente nos determinarían las funciones de cada unidad administrativa.

RESULTADOS: Esta parte consiste en la elaboración de un reporte escrito en donde se presentará los resultados obtenidos de la investigación que concluyen con la definición de la estructura organizacional de tipo funcional y la descripción de funciones de las áreas que integran la organización.

CONCLUSIONES: La investigación fue en colaboración con el personal, que labora en el CREST, aportando la descripción de sus actividades. Después de revisar los diferentes tipos y diseños de organigramas, se decidió por la implementación y diseñó de un organigrama con estructura organizacional de tipo funcional, considerando el tipo de organismo social investigado y las cadenas de mando y canales de comunicación.

PALABRAS CLAVE: Organizaciones. Estructura organizacional. Definición de funciones.

\section{ABSTRACT}

OBJECTIVE: To analyze the organizational structure of the Social Readaptation Center in Tabasco (SRCT).

MATERIAL AND METHOD: The research was carried out in an institution that depends directly from the public administration. In a first moment, an interview to the responsable person of the institution was performed. In a second moment, the administrative personnel was interviewed. An structured interview was used for all the informants in order to obtain a defined information about their activities later on determined by the functions of every administrative unit. 
ANÁLISIS DE LA ESTRUCTURA ORGANIZACIONAL DEL CENTRO DE REINSERCIÓN SOCIAL DE TABASCO (CREST).

RESULTS: This section consists of a written report where the results obtained from the research will be presented and it is concluded that the organizational structure is of functional type as well as the description of functions of the integrating areas.

CONCLUSIONS: The research was a collaborative one, with the assistance of the SRCT personnel, for the activities description. After analyzing the different organizational types and designs, an organigram of functional type was selected and designed to be implemented, considering the activities of this social organization being studied, as well as the chain of command and communication channels.

KEY WORDS: Organizations, Organizational Structure. Definitions of functions.

\section{INTRODUCCIÓN}

El diseño de una estructura organizacional adecuada contribuye a mejorar la competitividad. En las organizaciones pública o privada, lucrativa o no lucrativa es necesario definir las diferentes áreas funcionales que se desarrollan para la consecución de los objetivos establecidos en el diseño de la estructura organizacional interna que establece formalmente los niveles de autoridad y responsabilidad. La división en áreas funcionales y el diseño de la estructura, debe tener flexibilidad suficiente como para posibilitar una administración dinámica y basada en proyectos.

Por esto, la importancia de realizar un análisis de la estructura organizacional del Centro de Reinserción Social de Tabasco (CREST), con la finalidad de conocer si las responsabilidades, funciones y tareas de los empleados que se encuentran descritas en la estructura organizacional se realizan de manera eficiente.

El presente trabajo, describe las distintas actividades que se llevaron a cabo durante la investigación en el CREST en las Unidades Administrativas, donde se realizó un análisis de la estructura organizacional, funciones y tareas que allí se realizan. 
ANÁLISIS DE LA ESTRUCTURA ORGANIZACIONAL DEL CENTRO DE REINSERCIÓN SOCIAL DE TABASCO (CREST).

\section{Desarrollo}

Problematización. Uno de los mayores tropiezos que se suelen encontrar en las organizaciones tiene su origen en el diseño organizacional. Por esta razón, resulta fundamental hacer esfuerzos conducentes a construir una estructura que en mejor forma refleje los propósitos del CREST.

El CREST fue constituido en el año 1973, y cuenta con 46 años de servicio. La necesidad surge al darse cuenta la administración del CREST que, en este tiempo, el Centro de Reinserción ha crecido y que su estructura organizacional ha evolucionado en su desarrollo; por lo tanto, este ya no refleja su realidad. De allí, que conjuntamente con la administración se halla llegado a la conclusión que la estructura organizacional del CREST necesite ser analizada y reestructurada (Trujillo, 1971).

Partiendo de este hecho, se plantea realizar un análisis organizacional que nos permita conocer este CREST de forma tanto horizontal como vertical y que basado en este análisis se pueda proponer una estructura organizacional más acorde a la realidad actual, de manera que pueda establecer a través de los diferentes procesos y funciones que se ejecutan al interior de la misma, las líneas de autoridad y responsabilidad, prevenir la falta de compromiso de los colaboradores, desordenes en los procesos, fallas de comunicación, y falta de sentido de pertenencia y pueda responder satisfactoriamente con las exigencias de CREST, que contenga los propósitos claros de : visión, misión, objetivos y valores. Una estructura organizacional facilita la aplicación exitosa de las habilidades de sus colaboradores, lo cual nos lleva a la pregunta de investigación: ¿la estructura del CREST se encuentra acorde con la realidad actual y las exigencias del entorno interno y externo? (Lenin, 2008).

Justificación. El proceso de crecimiento en la Institución requiere de una estructura organizacional con sustento legal y procesos normados que sostengan dicha estructura, de manera que contribuyan eficazmente al aumento y mejora de los directivos y colaboradores en su área laboral (Martínez, 2002).

Las organizaciones. Una organización puede definirse como dos o más personas que colaboran dentro de unos límites definidos para alcanzar una meta común. En esta definición están implícitas varias ideas: las organizaciones están compuestas por personas, las organizaciones subdividen el trabajo entre sus individuos y, las organizaciones persiguen metas compartidas (Hodge, 2001). 
ANÁLISIS DE LA ESTRUCTURA ORGANIZACIONAL DEL CENTRO DE REINSERCIÓN SOCIAL DE TABASCO (CREST).

Las organizaciones están compuestas por personas: esta variable es la que hace que la organización sea el sistema más complejo del universo. Cuando las personas trabajan juntas, es necesario tener en cuenta una serie de aspectos; por ejemplo, es preciso dividir el trabajo entre los individuos y, tal como se ha dicho, se ha de buscar personas que posean conocimientos y habilidades especializadas. Para ejercer coordinación y control, los miembros de la organización necesitan una estructura formal donde se especifiquen los roles, las responsabilidades y las relaciones existentes entre los miembros de la empresa. Las organizaciones poseen límites definidos; intentar definir las fronteras o límites de una empresa puede parecer, a primera vista, una tarea sencilla; por tanto, la pertenencia como miembro es la característica que define los límites de una organización (Gibson, 2009).

Existen dos enfoques para establecer los límites de una organización; el primero, es aquel que enfatiza a las personas y su pertenencia como miembros y el segundo, se centra en el lugar donde se realizan las distintas actividades laborales (Docplayer, 2019).

La última parte de la definición de organización, enfatiza la tendencia de estas a la búsqueda de metas determinadas. Las empresas no son solo una agrupación de personas temporales, sino que existen para lograr metas compartidas de carácter común. En conclusión, las organizaciones son sistemas humanos de cooperación y coordinación integrados dentro de límites definidos, con el fin de alcanzar metas compartidas. La estructura organizacional debe cumplir con dos cometidos para la organización; debe proporcionar una estructura de responsabilidades, las relaciones de subordinación y los grupos, asimismo, debe ofrecer mecanismos para vincular y coordinar los elementos organizacionales en un todo coherente. La estructura se refleja en el organigrama. Vincular la organización en un todo coherente requiere de usos de sistemas de información e instrumentos de vinculación además del organigrama (Daft, 2011).

El concepto de estructura refiere a las formas y modos de relación entre las partes que componen la organización, para un lugar y tiempo determinado. El objeto de las estructuras como diseño es definir las tareas, la autoridad, las funciones y responsabilidades y los nexos de comunicación entre sectores que componen la organización. Es una forma de planeamiento que busca articular los esfuerzos para alcanzar los propósitos de la organización, aprovechar las capacidades existentes y atender las demandas del medio. La estructura establece de manera formal y pública las áreas o funciones, los niveles (jerarquía) y las relaciones entre sectores (Etkin, pp. 126-128). 
ANÁLISIS DE LA ESTRUCTURA ORGANIZACIONAL DEL CENTRO DE REINSERCIÓN SOCIAL DE TABASCO (CREST).

La estructura es un reflejo de la forma en que la organización se propone lograr sus objetivos, los criterios con que se dividen las tareas (por producto, lugar, proceso), quiénes son los responsables, los alcances de sus decisiones (sus competencias) y los recursos asignados a las unidades o sectores; es una división, pero también una articulación. En su origen, la estructura es parte del diseño o planteamiento de actividades, es una norma y una guía, pero su eficacia requiere que sea ajustada a la realidad cotidiana; es decir, da idea de un orden de articulación entre tareas o funciones, es un orden que sigue uno o varios criterios, tal como dividir por productos, por funciones o por zonas geográficas. La idea de estructura, sugiere que los componentes no se mueven libremente, sino siguiendo ciertas normas o pautas de relación, se habla de la estructura para hacer referencia a una disposición de las tareas y funciones que está más allá de las opiniones o las preferencias personales, pensada en función del conjunto.

Con el término de estructura se designa la distribución y el orden de las diversas partes de un todo. La estructura no tiene partes, sino miembros de u todo orgánico, los componentes de una estructura se hallan relacionados unos con otros de modo que sólo pueden ser lo que son en su relación con los demás y por ella. La estructura de algo consiste en las interrelaciones relativamente estables de sus partes (Méndez, 1986, p. 100).

Definición de Estructura. Se define como la suma total de las formas en las que una organización divide su trabajo en diversas tareas, coordinándolas entre si posteriormente (Hodge, 2001).

Las organizaciones crean una estructura oficial conocida como organización formal, representada en el organigrama (ver figura 1). Las líneas que conectan cada punto muestran las relaciones jerárquicas que existen en las empresas. 
ANÁLISIS DE LA ESTRUCTURA ORGANIZACIONAL DEL CENTRO DE REINSERCIÓN SOCIAL DE TABASCO (CREST).

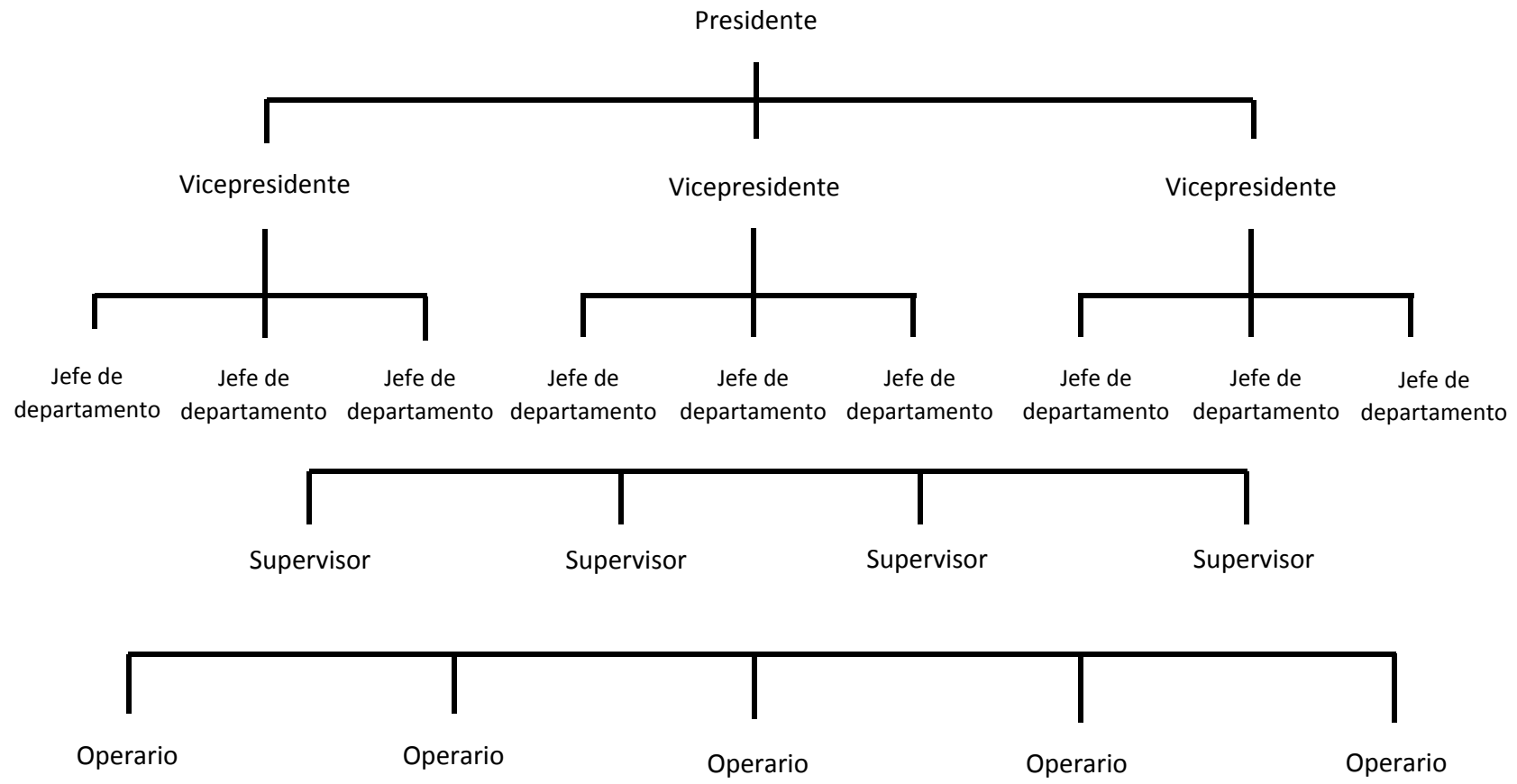

Figura 1. Tomado de (Mintzberg, 2002).

Fuente: Elaboración propia.

Como puede apreciarse en la figura 1, cada Jefe de departamento tendría un personal de supervisores similar al que viene representado, mientras que cada supervisor tendría un número determinado de subordinados. Cada nivel horizontal de puntos, representa un nivel de autoridad en la organización, por lo que todos los Jefes de departamento, en este caso, tendrían esencialmente el mismo nivel de autoridad. Un organigrama formal representa la estructura oficial autorizada explícitamente por la organización; esta estructura está constituida por las funciones y relaciones designadas formalmente, con independencia del individuo que ocupa esta función y de las personas con las que establece relaciones.

La estructura de la organización, puede definirse simplemente como el conjunto de todas las formas en que se divide el trabajo en tareas distintas, consiguiendo luego la coordinación de estas (Mintzberg, 2002).

Existen cinco mecanismos de control que explican las formas fundamentales en que las organizaciones coordinan su trabajo, las cuales son:

1. Adaptación mutua, consigue la coordinación del trabajo mediante la simple comunicación informal. El control de trabajo corre a cargo de los que lo realizan tal como se indica en la BANDA-IZETA M.B, BANDA-IZETA H., GUILLEMÍN-CALDERÓN H., JUÁREZ-SOLÍS S. RICO-MEZA J. H. 
ANÁLISIS DE LA ESTRUCTURA ORGANIZACIONAL DEL CENTRO DE REINSERCIÓN SOCIAL DE TABASCO (CREST).

figura 2, por tratarse de un sistema simple. La adaptación mutua, se utiliza como es natural, en las organizaciones más sencillas, como puedan ser dos personas en una canoa o unas cuantas en un taller de cerámica.

2. La supervisión directa, consigue la coordinación al responsabilizarse una persona del trabajo de los demás dándoles instrucciones y controlando sus acciones como puede observarse en la figura 2, de hecho, un cerebro único controla numerosas manos, como pueda ser el caso del supervisor del taller de cerámica o del que dicta con su voz el ritmo de los remos en la galera de guerra.

3. Normalización de los procesos de trabajo, se normalizan los procesos de trabajo cuando el contenido del mismo queda especificado; es decir, programado. Podría pensarse en las instrucciones del montaje incluidas en un juguete.

4. Normalización de los resultados, se normalizan los resultados al especificarse los mismos, como pueda darse con las dimensiones del producto o del rendimiento. No se le dice al taxista cómo debe conducir ni la ruta que debe tomar, sino simplemente le comunicamos nuestro destino.

5. Normalización de las habilidades, las habilidades (y los conocimientos), se normalizan cuando ha quedado especificado el tipo de preparación requerida para la realización del trabajo. Al encontrarse en el quirófano anestesistas y cirujano para una intervención de apendicitis, apenas necesitan comunicarse, puesto que, gracias a la preparación de ambos, saben exactamente lo que pueden esperar de la labor del otro; sus habilidades normalizadas se ocupan de gran parte de la coordinación. 
ANÁLISIS DE LA ESTRUCTURA ORGANIZACIONAL DEL CENTRO DE REINSERCIÓN SOCIAL DE TABASCO (CREST).

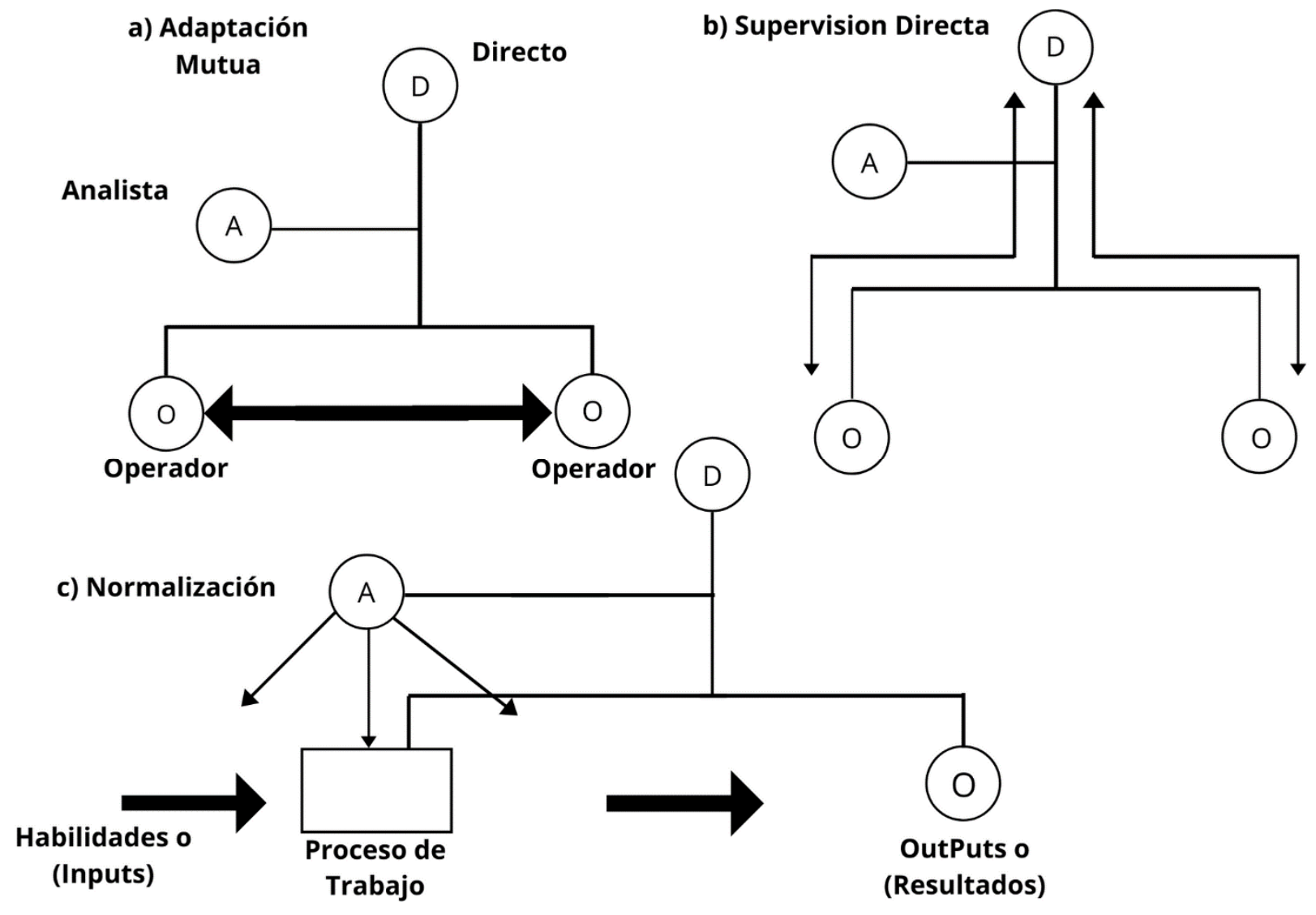

Figura 2. Tomado de (Mintzberg, 2002).

Fuente: Elaboración propia.

La importancia de la estructura organizacional, es el factor que influye en la conducta de los individuos y grupos que constituyen una organización, su importancia como fuente de influencia, está tan aceptada que algunos expertos definen el concepto como aquellas características de la organización que sirven para controlar o distinguir sus partes. La palabra clave en esta definición, es el control.

\section{Diagnóstico situacional}

EI CREST, se encuentra ubicado en el Km. 4.5 de la Carretera Villahermosa-Frontera, dentro de la periferia de la ciudad, en los márgenes de las Colonias Indeco y Ciudad Industrial. 
ANÁLISIS DE LA ESTRUCTURA ORGANIZACIONAL DEL CENTRO DE REINSERCIÓN SOCIAL DE TABASCO (CREST).

El sector geográfico de este Centro Penitenciario, es urbano, toda vez que cuenta con los servicios necesarios para la funcionalidad, como son: alumbrado, agua potable, drenaje y la seguridad de la zona.

La finalidad del CREST, es brindar la seguridad social a la población y el respeto a los derechos sociales mediante la rehabilitación y tratamiento a toda persona que infrinja las normas y leyes de conducta social, para cumplir este aspecto la prestación del servicio que brinde esta institución será de: seguridad, prevención, tratamiento y rehabilitación del individuo antisocial.

\section{MATERIAL Y MÉTODO}

La investigación se desarrolla en una institución dependiente de la administración pública. En primera instancia, se entrevista al titular de la dependencia; en segundo, se entrevista al personal administrativo. Para todos los entrevistados se utilizó una entrevista estructurada mediante una serie de preguntas, con la finalidad de obtener la información definida en sus actividades que posteriormente determinarían las funciones de cada unidad administrativa del CREST (Hernández, 2018).

Dada la naturaleza de la investigación, es de tipo exploratorio, ya que el objetivo es analizar las funciones que desempeñan las áreas de nivel directivo y operativo del CREST; además, se realizó un análisis e interpretación correcta sobre hechos y realidades de la situación actual de la institución (Bernal, 2006).

Asimismo, se llevó a cabo un censo a partir de todos los actores involucrados en las unidades administrativas, mismos que aportaron información útil para la investigación (Torres, 1971).

\section{RESULTADOS}

Los resultados obtenidos de la investigación, concluyen con la definición de la estructura organizacional de tipo funcional y la descripción de funciones de las áreas que integran la organización.

Asimismo, se detectó la inexistencia de una estructura orgánica definida, así como una identificación clara de los mandos jerárquicos, lo que confunde a los subordinados y por ende la falta orden jerárquico, provocando desorden al momento de ejecutar órdenes para realizar las 
ANÁLISIS DE LA ESTRUCTURA ORGANIZACIONAL DEL CENTRO DE REINSERCIÓN SOCIAL DE TABASCO (CREST).

funciones de cada puesto de trabajo y sin un manual de funciones los trabajadores no conocen cuáles son sus responsabilidades del CREST.

La definición de funciones a partir del Manual de Organizacional, dará una respuesta clara, compromiso y responsabilidad al personal que labora en el CREST, permitiendo con ello alcanzar de forma eficiente los objetivos a corto, mediano y largo plazo (Rodríguez, 2011).

\section{CONCLUSIONES}

El desarrollo de la investigación, fue en colaboración con el personal que labora en el CREST, aportando la descripción de sus actividades útiles y básicas, esenciales para el buen funcionamiento de la misma.

Después de revisar los diferentes tipos y diseños de organigramas, se decidió por la implementación y diseño de un organigrama con estructura organizacional de tipo funcional, considerando el tipo de organismo social investigado y las cadenas de mando y canales de comunicación.

Mediante la aplicación de la encuesta, se determinó la propuesta de la estructura organizacional y las funciones de los trabajadores, permitiendo con ello alcanzar los objetivos fijados, a partir de una visión más clara del todo en el CREST.

Aunado a la definición de la estructura organizacional, se definieron las funciones de las unidades administrativas, con la finalidad de procurarle a la institución el Manual de Organización, documento administrativo cuyo método describe las funciones y atribuciones que una institución, dependencia, empresa y demás organismos, necesitan para su buen funcionamiento y desempeño en el ámbito laboral. Además, de mantener una mejor organización, coordinación, ejecución y control de todas las actividades diarias que permitan al trabajador utilizar los recursos de una manera óptima con un rendimiento laboral alto.

\section{RECOMENDACIONES}

Considerando que los organigramas representan gráficamente la estructura organizacional de todo tipo de organismo, deben mostrar de igual forma la estructura jerárquica, los órganos que conforma la estructura y los canales de comunicación entre los órganos y los cargos. 
ANÁLISIS DE LA ESTRUCTURA ORGANIZACIONAL DEL CENTRO DE REINSERCIÓN SOCIAL DE TABASCO (CREST).

La representación de las relaciones de autoridad, deben ser dadas a partir de la autoridad de línea, ya que imparte las instrucciones a los subordinados, delegando con ello parte de su autoridad; asesoría o staff, además permite a las organizaciones tener personal para trabajos específicos o de asesoría en temas específicos; y la autoridad funcional, cuyo cargo determinado puede actuar sobre colaboradores no dependientes directamente, considerando de igual forma aspectos específicos de actividades en la organización.

En suma, un organigrama bien elaborado coadyuva a dejar claramente definidas las relaciones entre los diversos órganos de las entidades, instituciones, empresas o cualquier organismo del que se trate.

Por lo que refiere al Manual de Organización, es importante llevar a cabo las siguientes recomendaciones:

* Se recomienda dar a conocer el Manual de Organización al personal de nuevo ingreso, para que sepan cómo realizarán sus actividades y como deben desempeñarse dentro de su área laboral, buscando siempre el logro de los objetivos del CREST.

* Analizar y evaluar periódicamente los Manuales Administrativos, para establecer la correcta ejecución de las operaciones, con el fin de preservar la eficacia y eficiencia del CREST y se realicen las actualizaciones necesarias que deberán ser aprobadas por las autoridades pertinentes.

* Realizar las actualizaciones de común acuerdo con los empleados responsables de las áreas, cada vez, que lleguen a la conclusión de que un proceso determinado se debe modificar.

* Dar a conocer el Manual Organizacional y la estructura, con el fin de que el CREST, colaboradores y línea de supervisión, conozcan claramente lo que se requiere para cada puesto y las funciones a desempeñar por cada uno, de tal forma que exista responsabilidad y un alto sentido de cumplimiento, aprovechando y utilizando al máximo los recursos.

* Realizar cursos de sensibilización al personal que justifiquen el uso de los manuales administrativos previo a la publicación de los mismos.

* Ubicar al personal de acuerdo a su perfil profesional y funciones en las áreas correspondientes.

* Publicar el Manual de Organización del CREST, para que se oficialice la existencia del mismo como una Unidad Administrativa de vital importancia en el proceso de toma de decisiones. 
ANÁLISIS DE LA ESTRUCTURA ORGANIZACIONAL DEL CENTRO DE REINSERCIÓN SOCIAL DE TABASCO (CREST).

\section{REFERENCIAS BIBLIOGRÁFICAS}

Bernal, C. (2006). Metodología de la investigación. México: Pearson Prentice Hall.

Daft, R. (2011). Teoría y diseño organizacional. Mexico: CENGAGE.

Docplayer. (2019). Recuperado de https://docplayer.es/58752767-Universidad-simon-bolivarvicerrectorado-academico-decanato-de-estudios-tecnologicos-coordinacion-deadministracion-del-y-organizacion-empresarial.htML

Etkin, J. (2000). Política, gobierno y gerencia de las organizaciones. Buenos Aires, Argentina: Prentice Hall.

Gibson, I. y. (2009). Organizaciones: comportamiento, estructura y procesos. México, D.F.: McGraw-Hill.

Hernández, R. (2018). Metodología de la investigación. Las rutas cuantitativa, cualitativa y mixta. México: McGraw-Hill Education.

Hodge, B., Anthony, W. y Gales, L. (2001). Teoría de la organización, un enfoque estrategico. Madrid, España: Prentice Hall.

Lenin, M. P. (2008). Derecho penitenciario. México, D.F.: Oxford University Press México.

Madrazo, C. A. (1959). Primer informe de gobierno. Tabasco, México: Talleres Editoriales del Gobierno de Tabasco.

Martínez, G. M. (2002). Derecho de policía. México, D.F: Flores Editor y Distribuidor, S.A. de C.V.

Mendez, J., Monroy, F. y Zorrilla, S. (1986). Dinámica social de las organizaciones. México, D.F.: Interamericana.

Mintzberg, H. (2002). La estructuración de las organizaciones. Barcelona, España: Ariel.

Ramírez, M. L. (1997). Historia y Geografía de Tabasco, Asociación de Historiadores de Tabasco. Tabasco, México: Olkos.

Rodríguez, J. (2011). Cómo elaborar y usar los manuales administrativos. México, D.F.: Cengage Learning.

Torres, R. H. (s.f.). Metodología de la investigación. Las rutas cuantitativa, cualitativa y mixta. México, D.F.: McGraw-Hill Education.

Town.educ/security, G. (junio de 2007). Informe labores plataforma México. Obtenido de Informe Labores

Plataforma

México: http://pdba.georgetow.educ/security/citizensecurity/mexico/evaluaciones/informeLaboresplataformamexico.

Trujillo, M. (1971). Primer informe de gobierno. Tabasco, México: Talleres Editoriales del Gobierno de Tabasco. 